\title{
Adrenal crisis secondary to bilateral adrenal haemorrhage after hemicolectomy
}

\author{
Anthony Logaraj1,2, Venessa H M Tsang2,3, Shahrir Kabir1,2 and Julian C Y Ip1,2 \\ 1Department of Surgery, Royal North Shore Hospital, St Leonards, New South Wales, Australia, ${ }^{2}$ The University of Sydney, \\ Sydney, Australia, ${ }^{2}$ Department of Endocrinology, Royal North Shore Hospital, St Leonards, New South Wales, Australia
}

\author{
Correspondence \\ should be addressed \\ to A Logaraj \\ Email \\ anthony.logaraj@health.nsw. \\ gov.au
}

\section{Summary}

Adrenal haemorrhage is a rare cause of adrenal crisis, which requires rapid diagnosis, prompt initiation of parenteral hydrocortisone and haemodynamic monitoring to avoid hypotensive crises. We herein describe a case of bilateral adrenal haemorrhage after hemicolectomy in a 93-year-old female with high-grade colonic adenocarcinoma. This patient's post-operative recovery was complicated by an acute hypotensive episode, hypoglycaemia and syncope, and subsequent computed tomography (CT) scan of the abdomen revealed bilateral adrenal haemorrhage. Given her labile blood pressure, intravenous hydrocortisone was commenced with rapid improvement of blood pressure, which had incompletely responded with fluids. A provisional diagnosis of hypocortisolism was made. Initial heparin-induced thrombocytopenic screen (HITTS) was positive, but platelet count and coagulation profile were both normal. The patient suffered a concurrent transient ischaemic attack with no neurological deficits. She was discharged on a reducing dose of oral steroids with normal serum cortisol levels at the time of discharge. She and her family were educated about lifelong steroids and the use of parenteral steroids should a hypoadrenal crisis eventuate.

\section{Learning points:}

- Adrenal haemorrhage is a rare cause of hypoadrenalism, and thus requires prompt diagnosis and management to prevent death from primary adrenocortical insufficiency.

- Mechanisms of adrenal haemorrhage include reduced adrenal vascular bed capillary resistance, adrenal vein thrombosis, catecholamine-related increased adrenal blood flow and adrenal vein spasm.

- Standard diagnostic assessment is a non-contrast CT abdomen.

- Intravenous hydrocortisone and intravenous substitution of fluids are the initial management.

- A formal diagnosis of primary adrenal insufficiency should never delay treatment, but should be made afterwards.

\section{Background}

Bilateral adrenal haemorrhage is a particularly uncommon occurrence especially in a patient with no previous adrenal pathology. It is most commonly seen in the first fortnight postoperatively $(1,2)$. Rapid diagnosis should be made with a non-contrast CT abdomen scan. Delay in diagnosis can lead to adrenal crisis. The pathogenesis of adrenal haemorrhage is not yet fully elucidated but may include reduced adrenal vascular bed capillary resistance, adrenal vein thrombosis, catecholamine-related increased adrenal blood flow and adrenal vein spasm.

\section{Case presentation}

A 93-year-old female presented with two weeks of left upper quadrant abdominal pain associated with $10 \mathrm{~kg}$ unintentional weight loss. Pre-operative computed 
tomography (CT) scan demonstrated a splenic flexure mass with bowel obstruction. Her medical history included dementia, bladder transitional cell carcinoma without known metastases, laparoscopic cholecystectomy and bilateral knee replacement complicated by a provoked deep vein thrombosis. There was no other personal or family history of pro-thrombotic disorders. On examination, the patient was tender upon deep palpation in the left upper quadrant, but otherwise well and haemodynamically stable.

A midline laparotomy demonstrated a large obstructing mass in the mid-transverse colon. The colon was mobilised from sigmoid colon to the terminal ileum. The ileocolic and middle colic vessels were ligated with preservation of the left colic artery. The colon was transected at the distal descending colon and terminal ileum and a side-to-side stapled anastomosis was performed. The patient was managed in the intensive care unit for $24 \mathrm{~h}$. Histopathology confirmed a T3 highgrade adenocarcinoma measuring $14 \times 6.5 \mathrm{~cm}$, with 1 of 15 lymph nodes involved by carcinoma. Given her comorbidities and age, further treatment with chemotherapy was not pursued.

Her post-operative recovery was complicated on the fourth day with increasing abdominal pain and an acute hypotensive episode (systolic blood pressure $60 \mathrm{mmHg}$ ), hypoglycaemia of $3.2 \mathrm{mmol} / \mathrm{L}$ and syncope, which initially responded to fluid resuscitation, but recurred two hours later. Haemoglobin dropped from $96 \mathrm{~g} / \mathrm{L}$ to $89 \mathrm{~g} / \mathrm{L}$. An urgent CT scan to exclude post-operative haemorrhage was performed, which demonstrated acute bilateral adrenal haemorrhage with the right adrenal gland measuring $42 \times 33 \mathrm{~mm}$ and the left adrenal gland measuring $36 \times 30 \mathrm{~mm}$ (Figs 1 and 2). Review of previous imaging of the adrenal glands demonstrated no underlying pathology; size $28 \times 6 \mathrm{~mm}$ (right) and $25 \times 7 \mathrm{~mm}$ (left) (Fig. 3). There was no evidence of haemorrhage at the operative site.

\section{Investigation}

A serum cortisol level added to bloods performed $6 \mathrm{~h}$ before the syncopal episode at $12 \mathrm{pm}$ was $273 \mathrm{nmol} / \mathrm{L}(0600 \mathrm{~h})$ (normal range: 100-535), which is inappropriately low for a severely ill female after abdominal surgery. At the time of haemorrhage, her sodium and potassium were mildly low at $133 \mathrm{mmol} / \mathrm{L}$ (normal range: 135-145) and $3.4 \mathrm{mmol} / \mathrm{L}$ (normal range: 3.5-5.2) respectively, consistent with fluid resuscitation. A repeat cortisol was only performed after the administration of intravenous hydrocortisone

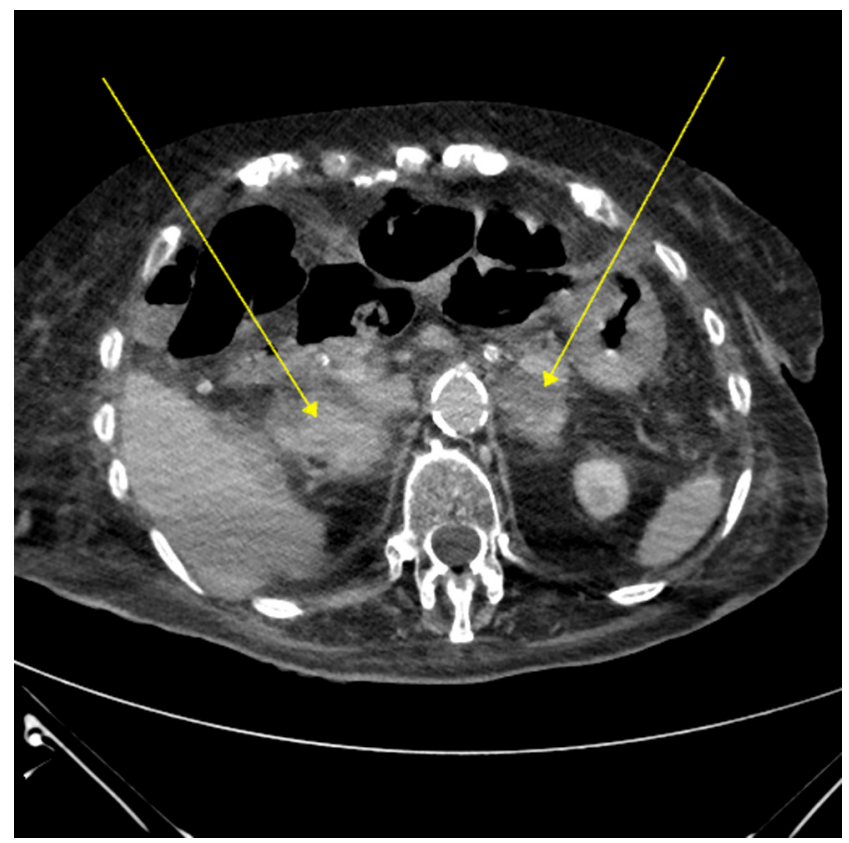

Figure 1

Axial computed tomography post-operatively with arrows indicating bilateral adrenal haemorrhage.

and was elevated at $1260 \mathrm{nmol} / \mathrm{L}(1500 \mathrm{~h})$, with an elevated ACTH $71 \mathrm{ng} / \mathrm{L}$ (reference range 7.2-63.3). Her calcium was $2.25 \mathrm{mmol} / \mathrm{L}$ (normal range: 2.1-2.6), creatinine $48 \mu \mathrm{mol} / \mathrm{L}$ (normal range: $45-90$ ) and glucose

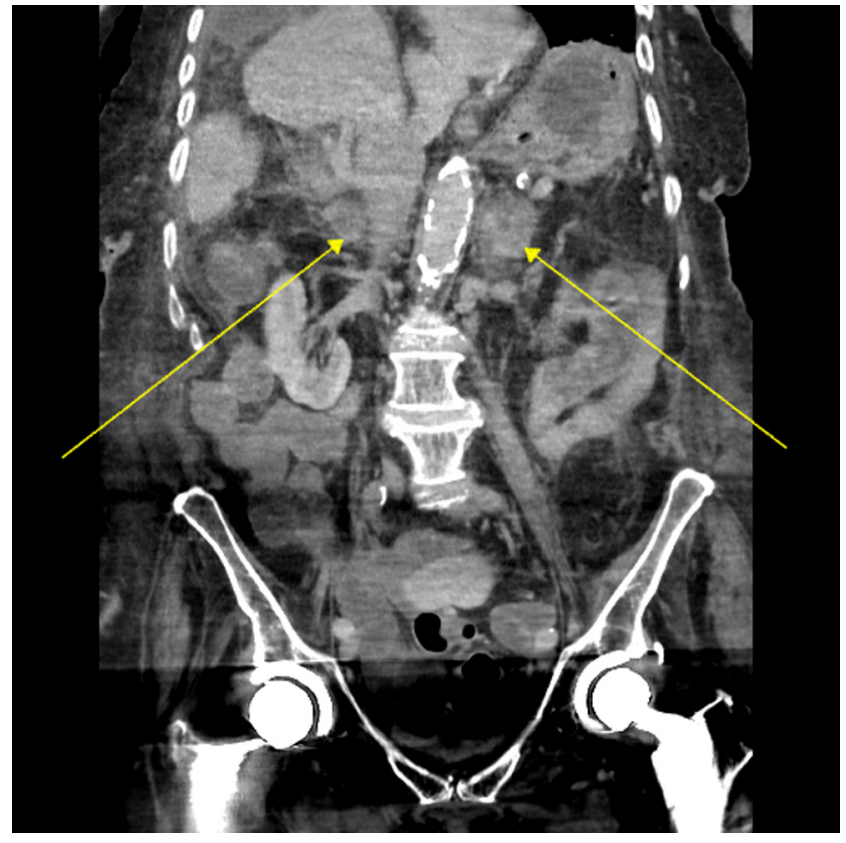

Figure 2

Coronal computed tomography post-operatively with arrows indicating bilateral adrenal haemorrhage. 


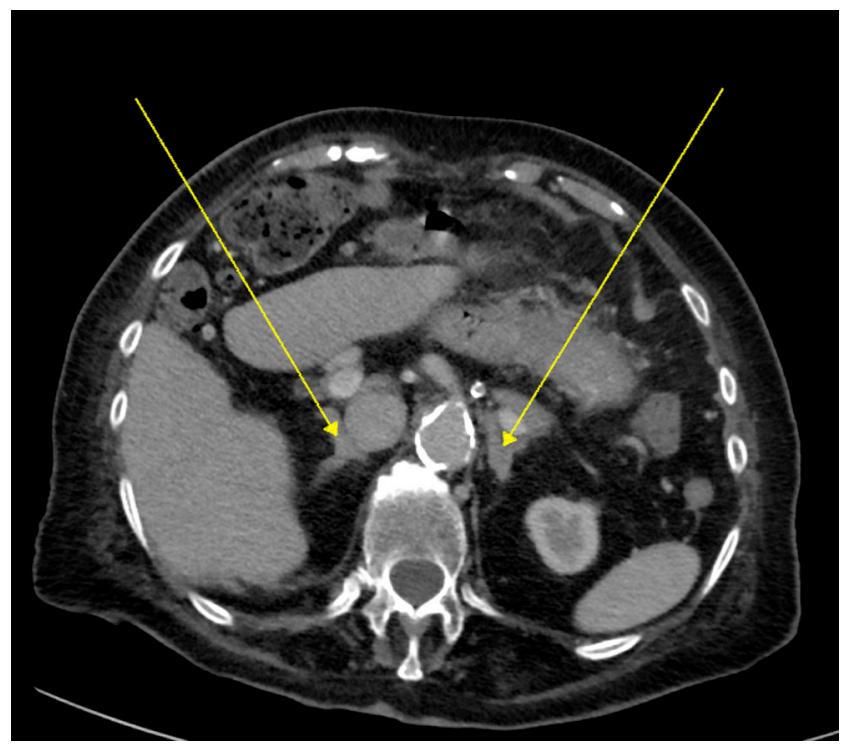

Figure 3

Axial computed tomography pre-operatively with arrows indicating the adrenal glands.

$6.2 \mathrm{mmol} / \mathrm{L}$ (normal range: 4.4-7.8). A repeat ACTH level performed $24 \mathrm{~h}$ later had dropped to the normal range of $8.0 \mathrm{ng} / \mathrm{L}$ after adequate glucocorticoid replacement. A provisional diagnosis of hypocortisolism was made due to the clinical presentation, bilateral adrenal haemorrhage, hyponatraemia, inappropriately low cortisol before the acute episode and response of ACTH after glucocorticoid replacement. Unfortunately, at the time, a short Synacthen test could not be performed due to her clinical instability and comorbidities. The hyperpigmentation characteristic of primary adrenal insufficiency (2) was not seen due to the acute onset.

To investigate the cause of the haemorrhage, a heparin-induced thrombotic thrombocytopenic screen (HITTS) was organised as she had been on prophylactic subcutaneous heparin perioperatively. The heparinplatelet factor 4 antibody assay was positive, but her platelet count and coagulation profile were normal with pre-operative platelets 365 , post-operative platelets 331 and post-haemorrhage platelets 280 (normal range: $150-400 \times 10^{3}$ platelets/microlitre). There was no evidence of venous thrombosis at the site of the adrenal haemorrhage. A second confirmatory functional test using heparin-induced platelet aggregation was negative. A prothrombotic screen was not performed due to interference from the haemorrhage and post-operative state. Seven days post-operatively, the patient suffered a transient ischaemic attack (TIA) resulting in $24 \mathrm{~h}$ of right upper limb paralysis with full recovery. Progress CT scan six days after haemorrhage demonstrated no size increase of the adrenal haemorrhage.

\section{Treatment}

Given her labile blood pressure, intravenous hydrocortisone $100 \mathrm{mg}$ daily was commenced as initial intravenous fluid resuscitation was unable to maintain her blood pressure. Aspirin was recommenced ten days after haemorrhage due to TIA, and the patient continued on a reducing dose of oral steroids, discharging on daily cortisone acetate $37.5 \mathrm{mg}$ mane and $25 \mathrm{mg}$ at $1600 \mathrm{~h}$, with fludrocortisone $0.1 \mathrm{mg}$ daily for mineralocorticoid replacement. The patient had several further episodes of hypotension during her in-hospital admission when her hydrocortisone was reduced or withheld to perform repeat serum cortisol levels. She was given a presumptive diagnosis of hypocortisolism, and she and her family were educated on the importance of lifelong steroids including the provision of an emergency steroid card, management of sick days and use of parenteral steroids should she have a hypoadrenal crisis.

\section{Outcome and follow-up}

On discharge, the patient was haemodynamically stable. She was moved into a dementia-specific nursing home with follow-up booked in the outpatient endocrinology clinic. She continues on replacement doses of hydrocortisone orally and is followed up by her general practitioner with latest fasting morning cortisol $156 \mathrm{nmol} / \mathrm{L}$ and fasting ACTH $22.6 \mathrm{ng} / \mathrm{L}$.

\section{Discussion}

Bilateral adrenal haemorrhage is an uncommon cause of hypocortisolism. Adrenal haemorrhage is most commonly seen in surgical patients in the first fourteen post-operative days $(1,2)$. Factors include sepsis, heparin-induced thrombocytopenia, myocardial infarction, congestive heart failure and anti-phospholipid syndrome $(2,3)$. However, it is more commonly associated with trauma, meningococcemia (Waterhouse-Friderichsen syndrome), and anticoagulation use (2). The underlying mechanism is not yet fully elucidated but may include ageing-related reduced capillary resistance in the adrenal vascular bed (3); adrenal vein thrombosis in hypercoagulable states (1) and, stress-induced catecholamine increase leading 
to increased adrenal blood flow, adrenal vein spasm and platelet aggregation causing reperfusion and subsequent bleeding (4). The presentation is often non-specific, as a result of hypocortisolism and haemorrhage, including abdominal pain, nausea, vomiting, confusion and hypotension $(5,9)$. The hyperpigmentation characteristic of primary adrenal insufficiency is initially not present when the insufficiency is acute. Consequently, bilateral adrenal haemorrhage causing acute primary adrenal insufficiency can be difficult to diagnose. Standard diagnostic assessment is a non-contrast CT, which would demonstrate hyperdense adrenal enlargement (6). Biochemical confirmations of hypoadrenalism includes low cortisol and elevated ACTH, with hyponatraemia and hyperkalaemia also present (7). In such situations, hyponatraemia should prompt physicians to consider adrenal insufficiency and surgeons to ensure adequate pre-operative sodium levels. In primary adrenal insufficiency, all layers of the adrenal cortex are affected, resulting in decreased production of glucocorticoids, mineralocorticoids and adrenally derived androgens (8). Adrenal insufficiency due to haemorrhage is initially managed with intravenous hydrocortisone and fluids (8). Long-term patients require lifelong steroid replacement and sick day management. This case demonstrated that adrenal crisis after adrenal haemorrhage requires rapid diagnosis, prompt initiation of parenteral hydrocortisone and haemodynamic monitoring to avoid hypotensive crises.

\section{Declaration of interest}

The authors declare that there is no conflict of interest that could be perceived as prejudicing the impartiality of the research reported.

\section{Funding}

This research did not receive any specific grant from any funding agency in the public, commercial or not-for-profit sector
Patient consent

The consent for publication of this study was obtained from the daughter of the patient.

\section{Author contribution statement}

Dr A L wrote the first draft; Dr S K was the patient's surgeon; Dr V T and $\mathrm{Dr} \mathrm{J}$ I supervised the care of the patient. All co-authors reviewed and contributed to the final manuscript.

\section{References}

1 Tormos L \& Schandl C 2013 The significance of adrenal haemorrhage: undiagnosed Waterhouse-Friderichsen syndrome, a case series. Journal of Forensic Science $\mathbf{5 8}$ 1071-1074. (doi:10.1111/1556-4029.12099)

2 Rao R, Vagnucci A \& Amico J 1989 Bilateral massive adrenal haemorrhage: early recognition and treatment. Annals of Internal Medicine 110 227-235. (doi:10.7326/0003-4819-110-3-227)

3 Anton E 2009 Spontaneous idiopathic bilateral massive adrenal haemorrhage in an elderly woman. Journal of the American Geriatric Society 57 2376-2377. (doi:10.1111/j.1532-5415.2009.02579.x)

4 Caron P, Chabannier J, Cambus P, Fortenfant F, Otal P \& Suc J 1998 Definitive adrenal insufficiency due to bilateral adrenal haemorrhage and primary antiphospholipid syndrome. Journal of Clinical Endocrinology and Metabolism 83 1437-1439. (doi:10.1210/ jcem.83.5.4833)

5 Barnett AH, Espiner EA \& Donald RA 1982 Patients presenting with Addison's disease need not be pigmented. Postgraduate Medical Journal 58 690-692. (doi:10.1136/pgmj.58.685.690)

6 Hoe C, Legmann P, Luton JP, Chapuis Y \& Fayet-Bonnin P 1995 Spontaneous unilateral adrenal haemorrhage: computerized tomography and magnetic resonance imaging findings in 8 cases. Journal of Urology 154 1647-1651. (doi:10.1016/ S0022-5347(01)66738-7)

7 Bornstein SR, Allolio B, Arit W, Barthel A, Don-Wauchope A, Hammer GD, Husebye ES, Merke DP, Murad MH, Stratakis CA, et al. 2016 Diagnosis and treatment of primary adrenal insufficiency: an endocrine society clinical practice guideline. Journal of Clinical Endocrinology and Metabolism 101 364-389. (doi:10.1210/ jc.2015-1710)

8 Arlt W \& Allolio B 2003 Adrenal insufficiency. Lancet 361 1881-1893. (doi:10.1016/S0140-6736(03)13492-7)

9 Ogino J, Toda J, Onitsuka S \& Hashimoto N 2013 Idiopathic bilateral adrenal haemorrhage related to acute adrenal insufficiency. BMJ Case Reports. (doi: 10.1136/bcr-2013-009626)

Revised version received 26 September 2016 Accepted 6 October 2016 